\title{
Clinical application of rapid assay of interleukin-6 in influenza-associated encephalopathy
}

\author{
Mikage Nakamura ${ }^{\mathrm{a}}$, Gaku Yamanaka ${ }^{\mathrm{a}}$, Hisashi Kawashima ${ }^{\mathrm{a}, *}$, Yoshiaki Watanabe ${ }^{\mathrm{a}}$, Hiroaki Ioi ${ }^{\mathrm{a}}$, \\ Yasuyo Kashiwagi $^{\mathrm{a}}$, Kouji Takekuma ${ }^{\mathrm{a}}$, Akinori Hoshika ${ }^{\mathrm{a}}$, Mizuho Hayakawa ${ }^{\mathrm{b}}$ and Shigeru Suzuki ${ }^{\mathrm{b}}$ \\ ${ }^{a}$ Department of Paediatrics, Tokyo Medical University, Japan \\ ${ }^{\mathrm{b}}$ Department of Central Clinical Laboratory, Tokyo Medical University, Japan
}

\begin{abstract}
The characteristics of influenza-associated encephalopathy is the high mortality and nimble progress with coma which appears in general cases within 48 hours. Most of patients show no abnormalities in the standard blood checks on admission or in early stage. In this study we investigated if a rapid assay of interleukin (IL)-6 is useful in influenza-associated encephalopathy in early stages. The levels of IL-6 in patients with influenza-associated encephalopathy did not show any significant difference compared with those in patients with febrile convulsion and rotavirus-associated convulsion. However the levels of IL-6 in severe cases were significantly higher than those of mild cases with influenza-associated encephalopathy. Consequently the rapid assay of serum IL-6 is useful to evaluate and decide the therapies.
\end{abstract}

Keywords: Influenza-associated encephalopathy, cytokine storm, interleukin-6, rapid assay

\section{Introduction}

The number of patients with a new type of influenzaassociated encephalopathy is increasing in Japan and in the United States [2,5]. According to the demographics of the Ministry of Health, Labor and Welfare in 1998, influenza was the sixth cause of death in children aged 1 to 4 years-old next to congenital heart diseases, and was the first cause of death within a single disease. Since 1988 , more than 60 patients with influenza-associated encephalopathy have been reported in Japan annually, and there is no sign of cessation. Mortality reached $30 \%$ in 1998, and although the rate decreased to approximately $15 \%$ in 2002 , the ratio of patients who recovered without sequela remained less than half. The characteristics of the influenza-associated encephalopathy

\footnotetext{
* Corresponding author: Hisashi Kawashima, MD, \& Ph.D., Department of Paediatrics, Tokyo Medical University, 6-7-1 Nishishinjuku, Shinjuku-ku, Tokyo160-0023, Japan. Tel.: +8133342 6111; Fax: +8133344 0643; E-mail: hisashi@tokyo-med.ac.jp.
}

is the high mortality and nimble progress with coma which appears within 48 hours in most cases1). At the time of consultation, the standard blood tests shows normal data in many cases, and then a rapid rise of AST, LDH, CPK, creatinine and FDP and a decreased number of platelets can be seen after a short time, which reveals DIC and MOF [5,8]. Although the pathophysiology of influenza-associated encephalopathy is not known because of a lack of the virus in the central nervous system, recent works showed that high levels of cytokines were detected $[3,8,9]$, especially proinflammatory cytokines for example IL-6 and TNF-alfa are high in serum and central spinal fluid. However they didn't mention and compare the IL-6 in other convulsive diseases. According to those data a high cytokine storm is suspected to be the center of the pathophysiology. From these considerations high dose gamma-globulin, steroid pulse and plasma exchange treatments are special evidences based on the treatment of influenza-associated encephalopathy at present. Intensive care trials in combination with (1) anti-virus 
drugs, (2) high dose gamma-globulin, (3) steroid pulse therapy, (4) high dose AT III, (5) head cooling and (6) plasma exchange started since 2001 [6]. In this study we investigated if a rapid assay of interleukin (IL)- 6 is useful in influenza-associated encephalopathy.

\section{Subjects and materials}

Fifteen children with influenza-associated encephalopathy aged 1 to 11 years old were enrolled in this study, and the case and severity were defined as follows: altered consciousness or loss of consciousness without prompt recovery and (1) mild: 7 cases with normal resolution (2) severe: 8 cases with sequelae or death and/or cases with multiple organ failure (MOF) ( 2 mortal and 3 sequelae). Serum levels of IL-6 were assayed by chemoluminescence Enzyme Immunoassay (CLEIA) by using Lumipulse $f$ (Fujirebio Diagnostics Inc, Tokyo, Japan) which can be resulted within 30 minutes. As control we analyzed serum IL-6 from 9 patients with febrile convulsion and 5 patients with rotavirus enterocolitis-associated convulsion, and compared the clinical causes and other blood data. All 9 patients with febrile convulsion showed neither prolonged unconsciousness nor any abnormality on EEG and laboratory findings. All blood samples were subjected at the time of their admission because of convulsion or unconsciousness. Infections of influenza and rotavirus were diagnosed by viral antigen tests. The statistical analysis (Fisher's Protected Least Significant Difference) was one-sided, $P$ values that were less than 0.05 were considered to indicate statistical significance. Statistical analysis was performed with Statcel software (OMS, Saitama, Japan).

\section{Results}

At first the levels of WBC, LDH, CPK and CRP of patients with influenza-associated encephalopathy, febrile convulsion and rotavirus enterocolitisassociated convulsion were investigated. Within the 3 groups there was no significant difference, which is shown in Fig. 1. The levels of IL-6 in patients with influenza-associated encephalopathy did not show any significant difference compared with those in patients with febrile convulsion and rotavirus-associated convulsion, which is shown in Fig. 2. There was no significant difference in the levels of In addition we analyzed serum IL-6 in 10 patients with influenza with- out convulsion, and all were under the normal range (6.0 pg/ml).

We compared the IL- 6 and other clinical data (duration of unconsciousness or convulsion), the number of WBC and platelets, creatinin, AST, CPK and LDH in mild cases of influenza-associated encephalopathy with severe cases. There were significant differences in the duration of unconsciousness, CPK, LDH and IL-6, which is shown in Fig. 3. There was no significant difference in the levels of platelets, creatinin and AST. We also assayed the fluctuation of IL-6 in severe cases before and after therapies of plasma exchange and pulse methyprednisolone. IL-6 decreased spontaneously after therapies as course of recovery.

\section{Discussion}

IL-6 is a multifunctional cytokine that plays a central role in the host defense due to its wide range of immune and hematopoietic activities and its potent ability to induce an acute phase response. And overexpression of IL- 6 has been implicated in the pathology of a number of diseases including rheumatoid arthritis and so on [7]. Aiba measured serum IL-6, TNF-alpha, soluble TNF-receptor 1, interferon-gamma and IL-2 in 6 patients with influenza-associated encephalopathy by ELISA method, and reported that IL-6 is most useful for the diagnosis and the levels reflected the clinical condition [1]. Kawada also reported that the transcription of IL-6, IL-10 and TNF-alpha genes were up-regulated to a greater extent in patients with influenza-associated encephalopathy than in those with influenza without neurological complications [4]. There was no significant difference in the levels of CPK, LDH obtained from patients with influenza-associated encephalopathy and with those of other convulsive diseases. However there were significant differences in the duration of unconsciousness, CPK, LDH and IL-6 between severe and mild cases with influenza-associated encephalopathy. From those findings IL-6 is a good and useful disease marker to decide treatments in influenzaassociated encephalopathy. In all previous reports the ELISA method was used, which takes a minimum of 3.5 hours and needs many samples at once. In this study we used a rapid assay of IL-6 (CLEIA) which takes only 30 minutes to obtain results and can be done by one sample. Since the progression of influenzaassociated encephalopathy is very quick CIEIA is a recommended method. We analyzed serum IL-6 in influenza-associated encephalopathy and compared the 

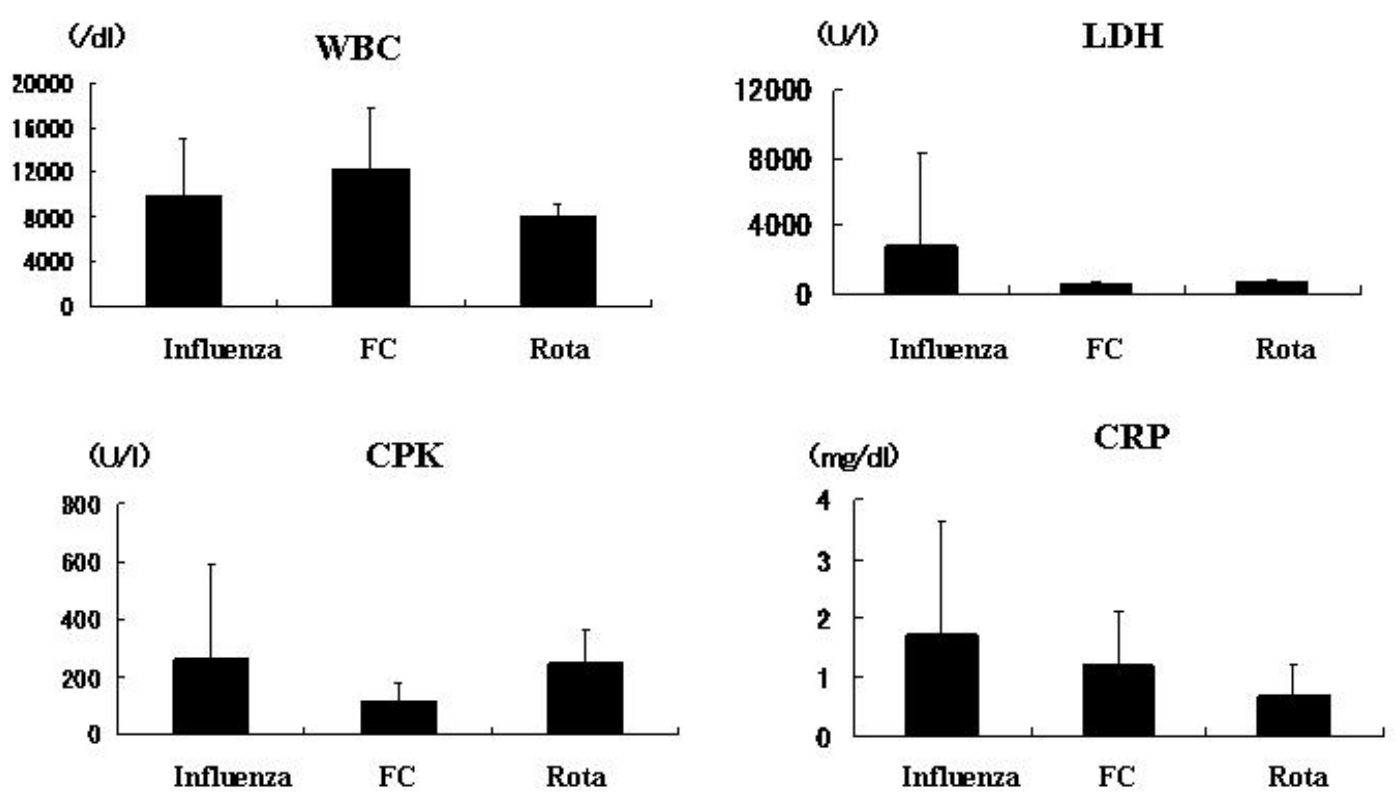

"Influenza" is influenza-associated encephalopathy, "FC" means febrile convlusion and "Rota" means rotavirus enteocolitis aosscoated convlusion.

Fig. 1. The comparison of duration of unconsciousness, CPK and LDH.

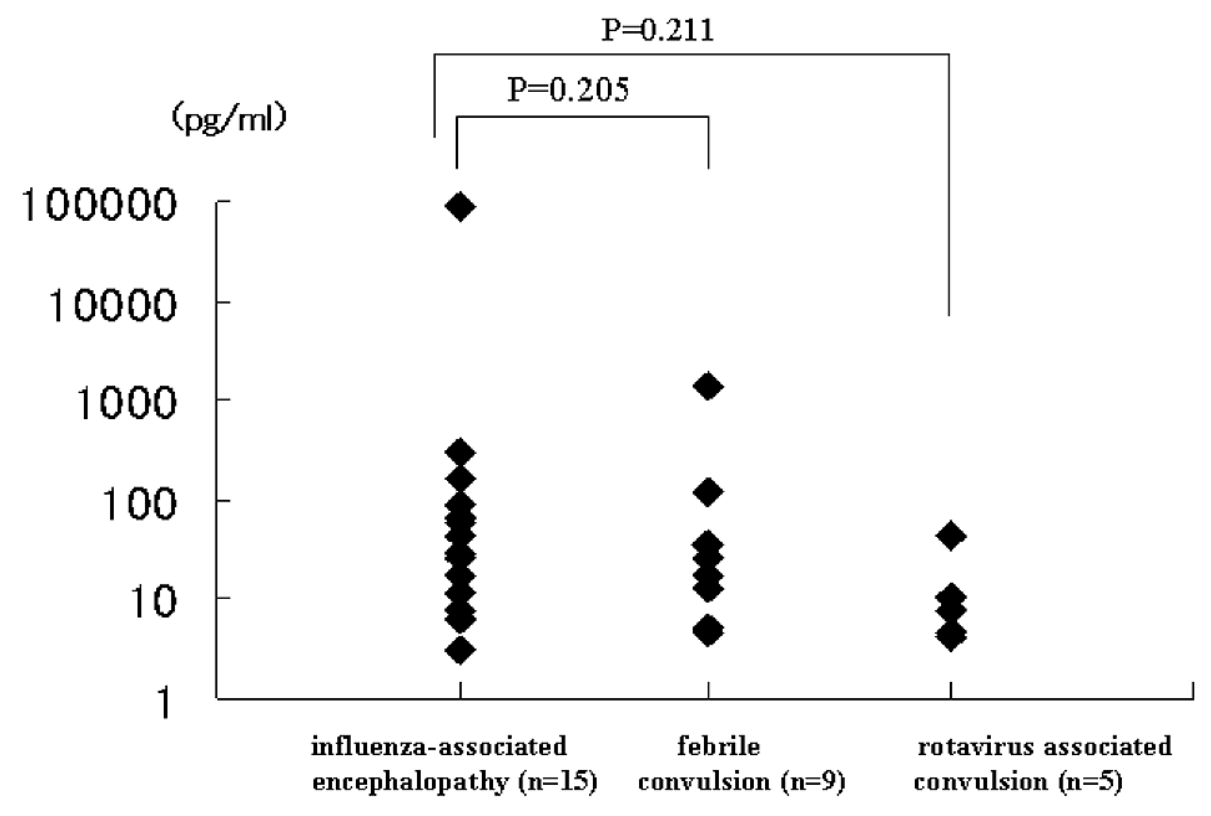

There was no significant difference within the 3 groups $(\mathrm{p}<0.05)$

Fig. 2. Serum IL-6 in influenza-associated encephalopathy and other diseases.

levels in patients with febrile convulsion and rotavirus enterocolitis-associated convulsion. The levels of some cases were higher than those of other diseases, how- ever statistically not significant. On the other hand serum IL-6 was a good marker to decide the severity of influenza-associated encephalopathy to prevent follow- 

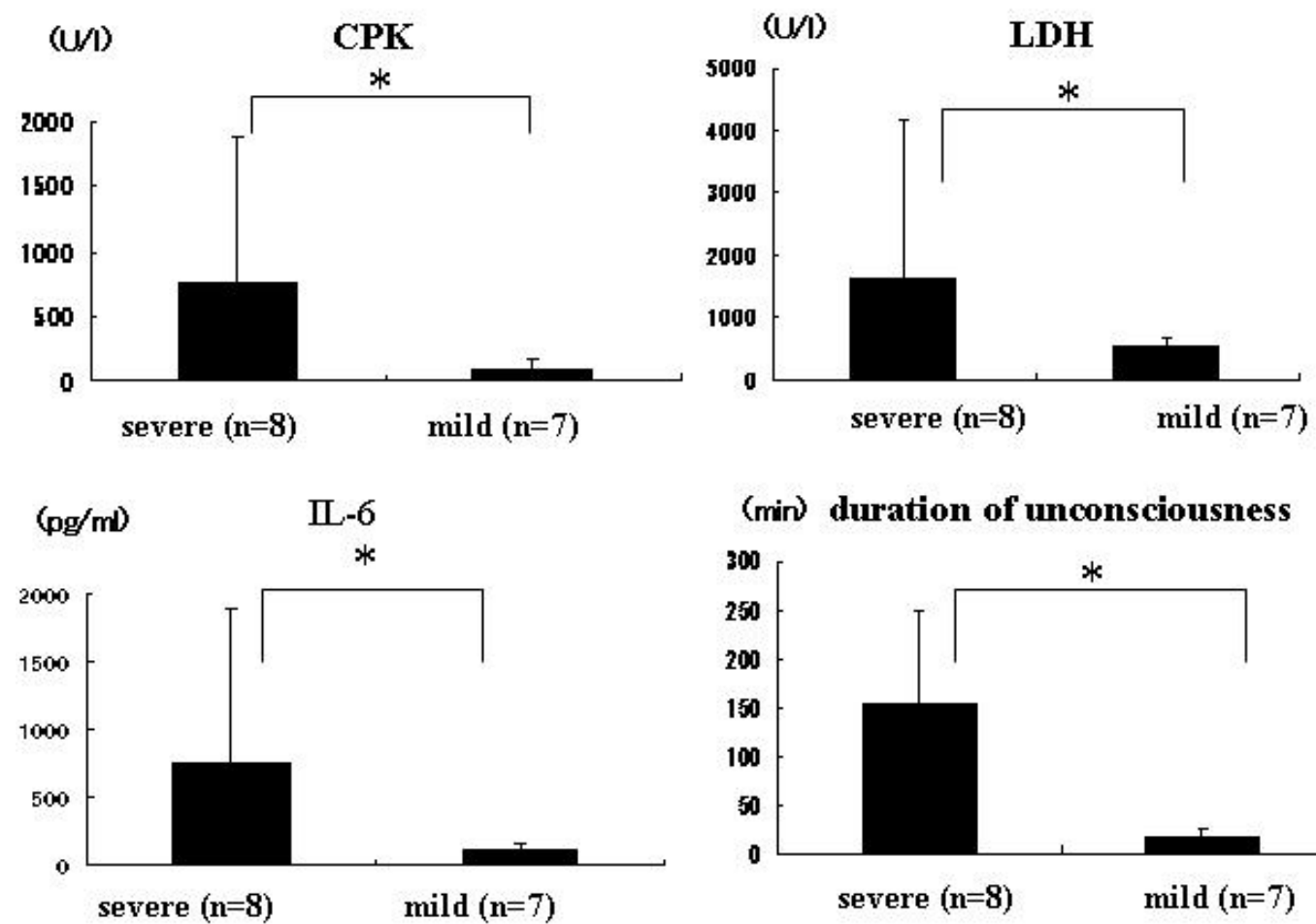

\section{(min) duration of unconsciousness}

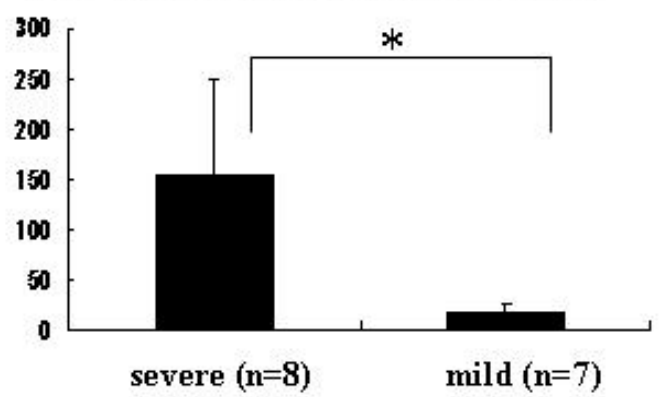

* significant differences within 2 groups $(\mathrm{p}<0.05)$

Fig. 3. Duration of unconsciousness, CPK, LDH and IL-6 within mild and severe patients with influenza-associated encephalopathy.

ing MOF. Consequently, a rapid assay of serum IL-6 would be a reliable method to decide therapies.

\section{Acknowledgment}

This study was supported by grants from the Ministry of Health, Labor and Welfare, Japan and Kawano Masanori Memorial Foundation for Promotion of Pediatrics.

\section{References}

[1] H. Aiba, M. Mochizuki, M. Kimura and H. Hojo, Predictive value of serum interleukin-6 level in influenza virus-associated encephalopathy, Neurology 57 (2001), 295-299.

[2] Centers for Disease Control and Prevention (CDC). Update: influenza-associated deaths reported among children aged $<18$ years-United States, 2003-04 influenza season, MMWR Morb Mortal Wkly Rep 52 (2004), 1286-1288.
[3] T. Ichiyama, H. Isumi, H. Ozawa, T. Matsubara, T. Morishima and S. Furukawa, Cerebrospinal fluid and serum levels of cytokines and soluble tumor necrosis factor receptor in influenza virus-associated encephalopathy, Scand J Infect Dis 35 (2003), 59-61.

[4] J. Kawada, H. Kimura, Y. Ito, S. Hara, M. Iriyama, T. Yoshikawa and T. Morishima, Systemic cytokine responses in patients with influenza-associated encephalopathy, J Infect Dis 188 (2003), 690-698.

[5] T. Morishima, T. Togashi, S. Yokota et al., Encephalitis and encephalopathy associated with influenza epidemic in Japan, Clin Inf Dis 35 (2002), 512-517.

[6] T. Morishima, [Treatment of influenza-associated encephalopathy], Nippon Rinsho 61 (2003), 2006-2012, Japanese.

[7] R.J. Simpson, A. Hammacher, D.K. Smith, J.M. Matthews and L.D. Ward, Interleukin-6: structure-function relationships, Protein Sci 6 (1997), 929-955.

[8] T. Togashi, Y. Matsuzono, M. Narita and T. Morishima, Influenza-associated acute encephalopathy in Japanese children in 1994-2002, Virus Res 103 (2004), 75-78.

[9] H. Yoshikawa, S. Yamazaki, T. Watanabe and T. Abe, Study on influenza-associated encephalitis/encephalopathy in children during the 1997 to 2001 influenza seasons, J Child Neurol 16 (2001), 885-890. 


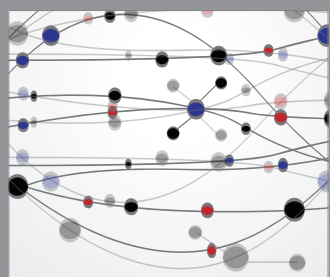

The Scientific World Journal
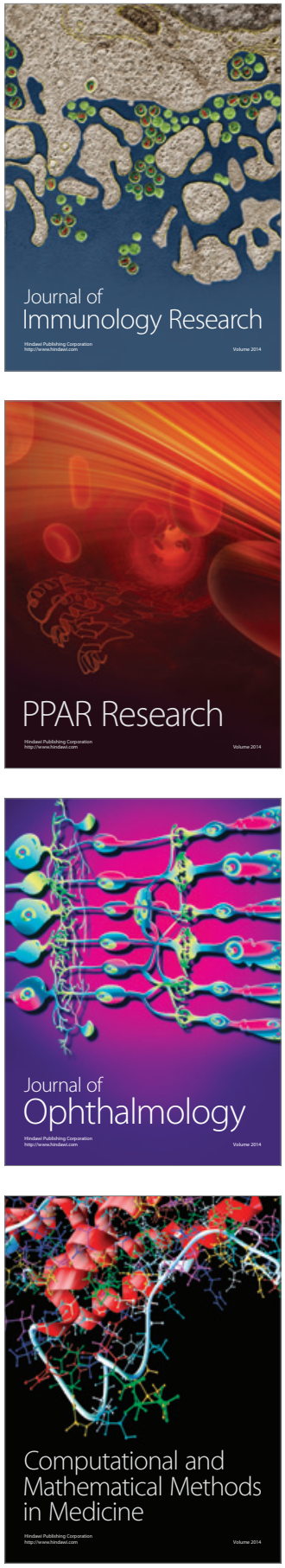

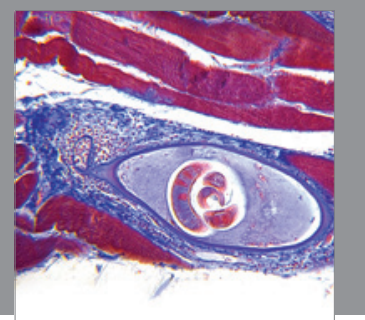

Gastroenterology

Research and Practice
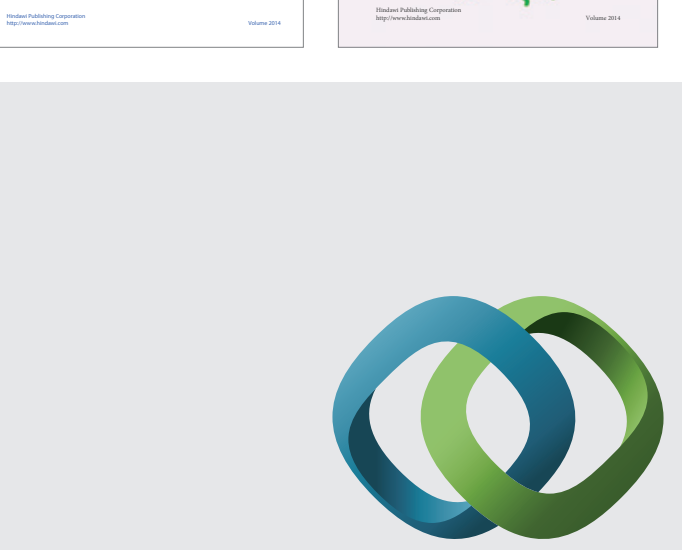

\section{Hindawi}

Submit your manuscripts at

http://www.hindawi.com
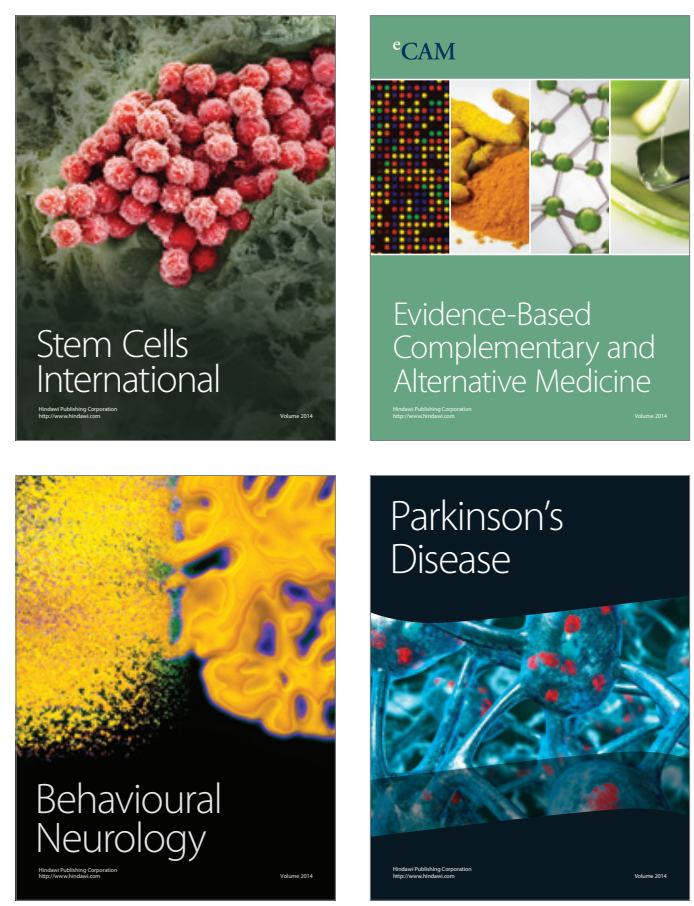

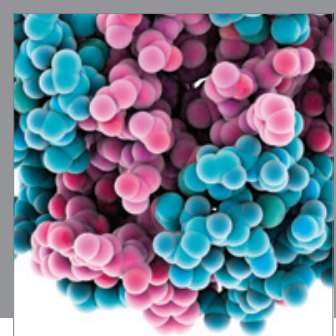

Journal of
Diabetes Research

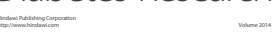

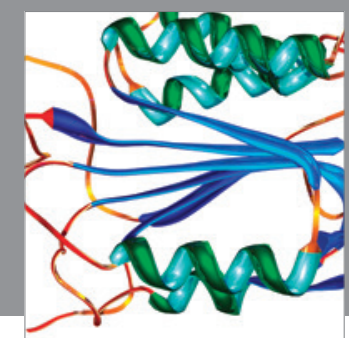

Disease Markers
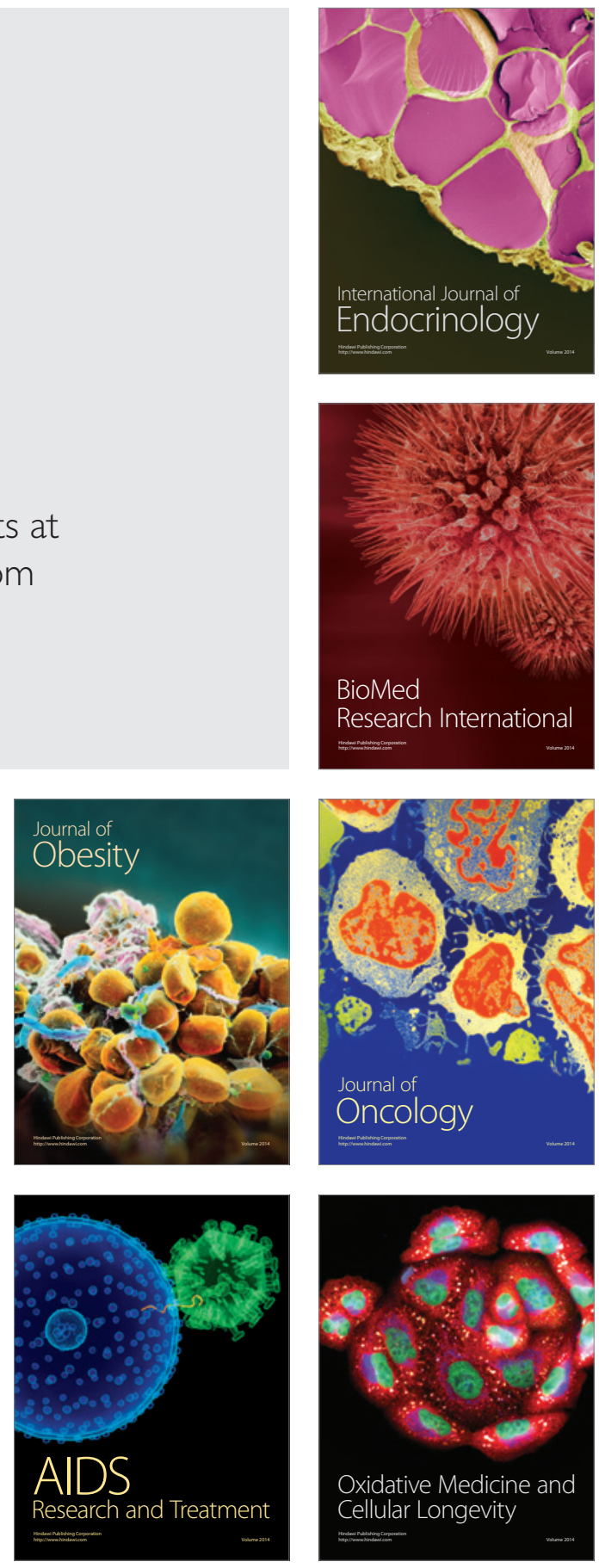